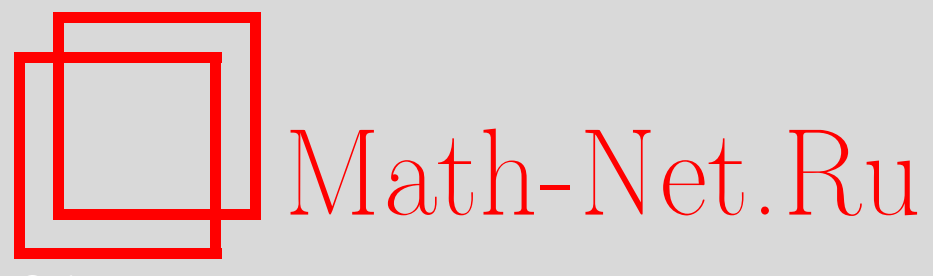

Н. М. Меженная, В. Г. Михайлов, О распределении числа единиц в выходной последовательности генератора Пола над полем $G F(2)$, Матем. вопр. криптогр., 2013, том 4, выпуск 4, 95-107

DOI: https://doi.org/10.4213/mvk101

Использование Общероссийского математического портала Math-Net.Ru подразумевает, что вы прочитали и согласны с пользовательским соглашением http://www.mathnet.ru/rus/agreement

Параметры загрузки:

IP : 54.210 .77 .194

26 апреля 2023 г., 11:58:13

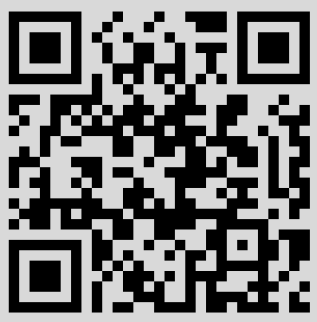




\section{О распределении числа единиц В Выходной последовательности генератора Пола над полем $\boldsymbol{G} \boldsymbol{F}(2)$

\author{
Н. М. Меженная ${ }^{1}$, В. Г. Михайлов ${ }^{2}$ \\ ${ }^{1}$ МГТУ им. Н. Э. Баумана, Москва \\ ${ }^{2}$ Математический институт им. В. А. Стеклова РАН, Москва
}

Получено 20.IV.2012

Доказаны оценки точности аппроксимации распределения числа единиц в полном цикле выходной последовательности генератора Пола над полем $G F(2)$ распределениями нормального типа. Выведены достаточные условия сходимости в соответствующих предельных теоремах.

Ключевые слова: мультициклический генератор Пола, нормальная аппроксимация, логарифмически нормальное распределение

On the distribution of the number of ones in the output sequence of the MCV-generator over $\boldsymbol{G} \boldsymbol{F}(2)$

\section{N. M. Mezhennaya ${ }^{1}$, V. G. Mikhailov ${ }^{2}$ \\ ${ }^{1}$ Bauman State Technical University, Moscow \\ ${ }^{2}$ Steklov Mathematical Institute of RAS, Moscow}

Abstract. For the distribution of the number of ones in the whole cycle of the output sequence of the MCV-generator over the field $G F(2)$ we obtain estimates of the accuracy of normal-like approximations.

Key words: the MCV-generator, the normal approximation, the log-normal distribution

Citation: Mathematical Aspects of Cryptography, 2013, vol. 4, no. 4, pp. 95-107 (Russian). 


\section{1. Введение}

В работе П. Пола [1] был предложен генератор псевдослучайных чисел, состоящий из $r$ циклических регистров сдвига взаимно простых длин $m_{1}, \ldots, m_{r}$ над кольцом вычетов по некоторому модулю $M$. Обозначим через $\left(x_{0}^{(k)}, \ldots, x_{m_{k}-1}^{(k)}\right), k=1, \ldots, r$, векторы заполнений ячеек регистров. Будем использовать в индексах обозначение $t(m)=t \bmod m$. Знаки выходной последовательности генератора образуются по формуле

$$
z_{t}=x_{t\left(m_{1}\right)}^{(1)}+\ldots+x_{t\left(m_{r}\right)}^{(r)} \bmod M .
$$

В настоящей работе мы рассматриваем случай, когда $M=2$, а заполнения всех $r$ регистров представляют собой независимые наборы независимых случайных величин, имеющих равномерное распределение на множестве $\{0,1\}$. Исследуется распределение числа единиц $\xi_{r}$ на отрезке длины $L=m_{1} m_{2} \ldots m_{r}$ выходной последовательности генератора из $r$ регистров.

В работе получены:

1) Представление распределения величины $\xi_{r}$ в виде распределения произведения $r$ независимых случайных величин, имеющих биномиальные распределения.

2) Оценки точности аппроксимации распределения величины $\xi_{r}$ распределением произведения $r$ независимых случайных величин, имеющих стандартное нормальное распределение, и условия сходимости распределения величины $\xi_{r}$ к такому распределению при $m_{1}, \ldots, m_{r} \rightarrow \infty, r=$ const.

3) Оценки точности аппроксимации распределения величины $\xi_{r}$ логнормальным распределением и условия сходимости распределения величины $\xi_{r}$ к этому распределению при $m_{1}, \ldots, m_{r}, r \rightarrow \infty$.

В меньшей степени изучен случай, когда число регистров генератора растет, а длины некоторых из них могут оставаться фиксированными. В этом случае удалось лишь указать условия сходимости распределения величины $\xi_{r}$ к логнормальному распределению.

\section{2. Формулировки}

Рассмотрим генератор с $r$ регистрами взаимно простых длин $m_{1}, \ldots, m_{r}$. Пусть $X^{(i)}=\left(X_{1}^{(i)}, \ldots, X_{m_{i}}^{(i)}\right)-$ случайное заполнение $i$-го регистра. Все случайные величины в наборах $X^{(1)}, \ldots, X^{(r)}$ независимы в совокупности и имеют равномерное распределение на множестве $\{0,1\}$. 
Нас интересуют свойства распределения числа $\xi_{r}$ единиц в выходной последовательности генератора

$$
Z_{t}=\left(X_{t\left(m_{1}\right)}^{(1)}+\ldots+X_{t\left(m_{r}\right)}^{(r)}\right) \quad \bmod 2, \quad t=0,1, \ldots, L-1
$$

Условимся использовать обозначение $\mathcal{L}(W)$ для распределения случайной величины $W$, обозначение $\mathrm{B}(m)$ - для распределения числа успехов в равновероятной последовательности Бернулли длины $m$, центрированного своим математическим ожиданием $m / 2$ и нормированного корнем из дисперсии $\sqrt{m} / 2$, и обозначение $\mathrm{B}\left(m_{1}, \ldots, m_{r}\right)$ - для распределения произведения $r$ независимых случайных величин, имеющих распределения $\mathrm{B}\left(m_{1}\right), \ldots, \mathrm{B}\left(m_{2}\right)$.

Теорема 1. Пусть $r \geq 2$, числа $m_{1}, \ldots, m_{r}$ взаимно просты, а случайные величины в наборах $X^{(1)}, \ldots, X^{(r)}$ независимы в совокупности и имеют равномерное распределение на множестве $\{0,1\}$. Тогда

$$
\mathcal{L}\left(\frac{2 \xi_{r}-m_{1} \ldots m_{r}}{\sqrt{m_{1} \ldots m_{r}}}\right)=\mathrm{B}\left(m_{1}, \ldots, m_{r}\right) .
$$

Следствие 1. Пусть выполнены условия теоремы 1. Тогда

$$
\begin{gathered}
\mathbf{E} \xi_{r}=\frac{m_{1} \ldots m_{r}}{2}, \quad \mathbf{D} \xi_{r}=\frac{m_{1} \ldots m_{r}}{4}, \\
\mathbf{E}\left(\xi_{r}-\mathbf{E} \xi_{r}\right)^{4}=\frac{1}{16} \prod_{i=1}^{r} m_{i}\left(3 m_{i}-2\right), \\
\mathbf{E}\left(\xi_{r}-\mathbf{E} \xi_{r}\right)^{2 k+1}=0, \quad k=1,2 \ldots, \\
\mathbf{E}\left(\xi_{r}-\frac{m_{1} m_{2} \ldots m_{r}}{2}\right)^{2 k}=2^{2 k(r-1)} \prod_{j=1}^{r} \mu\left(m_{j}, 2 k\right), \quad k=1,2, \ldots,
\end{gathered}
$$

где $\mu\left(m_{j}, k\right)=\left.\frac{1}{2^{m_{j}}} \frac{d^{k}}{d t^{k}}\left(1+e^{t}\right)^{m_{j}} e^{-t m_{j} / 2}\right|_{t=0}-k$-й центральнылй момент биномиального распределения с параметрами $m_{j} u 1 / 2$.

Равенство (3) показывает, что от $\xi_{r}$ полезно перейти к изучению величины

$$
\widetilde{\xi}_{r}=\frac{2 \xi_{r}-m_{1} \ldots m_{r}}{\sqrt{m_{1} \ldots m_{r}}} .
$$


Будем использовать обозначение $F_{X}$ для функции распределения случайной величины $X$, обозначение $\Phi$ для стандартной нормальной функции распределения и обозначение $\Phi_{r}$ для функции распределения произведения $r$ независимых случайных величин, имеющих стандартное нормальное распределение. Тогда $\Phi_{1}=\Phi$. Введем еще обозначение $d(U, V)$ для расстояния в равномерной метрике между функциями распределения $U$ и $V$ на прямой:

$$
d(U, V)=\sup _{-\infty<y<\infty}|U(y)-V(y)| .
$$

Теорема 2. Пусть $r \geq 2$, числа $m_{1}, \ldots, m_{r}$ нечетны, взаимно просты, а случайные величины в наборах $X^{(1)}, \ldots, X^{(r)}$ независимы в совокупности и имеют равномерное распределение на множестве $\{0,1\}$. Тогда

$$
d\left(F_{\vec{\xi}_{r}}, \Phi_{r}\right) \leq C_{B E} \sum_{i=1}^{r} \frac{1}{\sqrt{m_{i}}},
$$

где $C_{B E}$ - константа из неравенства Берри-Эссеена для распределения суммы независимых одинаково распределенных случайных величин.

ЗАмечанИЕ 1. Для константы $C_{B E}$ из неравенства Берри-Эссеена (см., например, [2]) для распределения суммы независимых одинаково распределенных случайных величин, обладающих нулевыми средними и конечными третьими абсолютными моментами, в настоящее время доказана оценка $C_{B E}<0,4774$ (см. [3]).

Следствие 2. Пусть выполнены условия теоремы $2, m_{1}, \ldots, m_{r} \rightarrow \infty$, a $r=$ const. Тогда при всех $-\infty<y<\infty$

$$
F_{\widetilde{\xi}_{r}}(y) \rightarrow \Phi_{r}(y)
$$

Нам удобно еще раз преобразовать исследуемую случайную величину $\xi_{r}$. Пусть

$$
\begin{gathered}
a=\sqrt{\frac{2}{\pi}} \int_{0}^{\infty} \ln x e^{-x^{2} / 2} d x=-0,6352 \ldots \\
\sigma^{2}=\sqrt{\frac{2}{\pi}} \int_{0}^{\infty}(\ln x-a)^{2} e^{-x^{2} / 2} d x=1,2337 \ldots \\
c=\sqrt{\frac{2}{\pi}} \int_{0}^{\infty}|\ln x-a|^{3} e^{-x^{2} / 2} d x=2,9607 \ldots
\end{gathered}
$$


Если $\eta$ имеет стандартное нормальное распределение, то $a=\mathbf{E} \ln |\eta|, \sigma^{2}=$ $=\mathbf{D} \ln |\eta|, c=\mathbf{E}|\ln | \eta|-a|^{3}$.

Положим

$$
\zeta_{r}=e^{-\frac{a}{\sigma} \sqrt{r}}\left|\widetilde{\xi}_{r}\right|^{\frac{1}{\sigma \sqrt{r}}}=e^{-\frac{a}{\sigma} \sqrt{r}}\left(\frac{\left|2 \xi_{r}-m_{1} \ldots m_{r}\right|}{\sqrt{m_{1} \ldots m_{r}}}\right)^{\frac{1}{\sigma \sqrt{r}}} .
$$

Введем обозначение $\Upsilon$ для функции стандартного логарифмически нормального распределения. Имеем

$$
\Upsilon(x)=\frac{1}{\sqrt{2 \pi}} \int_{0}^{x} \frac{1}{y} e^{-\ln ^{2} y / 2} d y .
$$

Теорема 3. Пусть выполнены условия теоремьл 2. Тогда

$$
d\left(F_{\zeta_{r}}, \Upsilon\right) \leq C_{B E}\left(2 \sum_{i=1}^{r} \frac{1}{\sqrt{m_{i}}}+\frac{c}{\sigma^{3} \sqrt{r}}\right)
$$

ЗАМЕЧАНИЕ 2. Если подставить в оценку (15) численные значения констант, приведенные в замечании 1 и формулах (11)-(13), то (15) преобразуется к виду

$$
d\left(F_{\zeta_{r}}, \Upsilon\right) \leq 0,96\left(\sum_{i=1}^{r} \frac{1}{\sqrt{m_{i}}}+\frac{2,27}{\sqrt{r}}\right)
$$

Воспользуемся теоремой 3 для описания свойств величин $\widetilde{\xi}_{r}$ и $\xi_{r}$.

Следствие 3. Пусть выполнень условия теоремы 2. При $-\infty<x<\infty$

$$
\begin{aligned}
\mid F_{\widetilde{\xi}_{r}}(x)- & \frac{1}{2}\left(1+\operatorname{sign}(x) \Phi\left(\frac{\ln |x|}{\sigma \sqrt{r}}-\frac{a}{\sigma} \sqrt{r}\right)\right) \mid \leq \\
\leq & C_{B E}\left(\sum_{i=1}^{r} \frac{1}{\sqrt{m_{i}}}+\frac{c}{2 \sigma^{3} \sqrt{r}}\right),
\end{aligned}
$$

где $\operatorname{sign}(x)=x /|x|$, если $x \neq 0, u \operatorname{sign}(0)=0, \Phi(-\infty)=0$. 
Следствие 4. Пусть выполнены условия теоремы 2. Тогда при $0.5<$ $\alpha<1$

$$
\begin{gathered}
\mathbf{P}\left\{\xi_{r}<\frac{1}{2}\left(m_{1} \ldots m_{r}+e^{a r+u_{2 \alpha-1} \sigma \sqrt{r}} \sqrt{m_{1} \ldots m_{r}}\right)\right\} \rightarrow \alpha, \\
\mathbf{P}\left\{\xi_{r}<\frac{1}{2}\left(m_{1} \ldots m_{r}-e^{a r+u_{2 \alpha-1} \sigma \sqrt{r}} \sqrt{m_{1} \ldots m_{r}}\right)\right\} \rightarrow 1-\alpha,
\end{gathered}
$$

где $u_{2 \alpha-1}-$ квантиль уровня $2 \alpha-1$ стандартного нормального закона распределения, определяемая уравнением $\Phi\left(u_{2 \alpha-1}\right)=2 \alpha-1$.

Наконец, рассмотрим более общий случай, когда длины некоторых регистров при переходе к пределу могут оставаться фиксированными.

Теорема 4. Пусть $r \rightarrow \infty$, при каждом $r$ числа $m_{1}, \ldots, m_{r}$ взаимно просты, $3 \leq m_{1}<m_{2}<\ldots<m_{r}$, а случайные величины в наборах $X^{(1)}, \ldots, X^{(r)}$ независимы в совокупности и имеют равномерное распределение на множестве $\{0,1\}$. Тогда при всех $0<y<\infty$

$$
\mathbf{P}\left\{\left|\widetilde{\xi}_{r}\right|<e^{\mathbf{E} \ln \left|\widetilde{\xi}_{r}\right|} y^{\sigma \sqrt{r}}\right\} \rightarrow \Upsilon(y) .
$$

ЗАМЕЧАНИЕ 3 . Если $r, m_{1}, \ldots, m_{r} \rightarrow \infty$ так, что

$$
\frac{1}{\sqrt{r}} \sum_{j=1}^{r} \frac{\ln m_{j}}{\sqrt{m_{j}}} \rightarrow 0,
$$

то $\mathbf{E} \ln \left|\widetilde{\xi}_{r}\right|=a r+o(\sqrt{r})$, а соотношение $(20)$ в теореме 4 принимает вид

$$
\mathbf{P}\left\{\left|\widetilde{\xi}_{r}\right|<e^{a r} y^{\sigma \sqrt{r}}\right\} \rightarrow \Upsilon(y) \quad \text { при всех } \quad 0<y<\infty .
$$

При условии

$$
\sum_{j=1}^{r} \frac{1}{\sqrt{m_{j}}} \rightarrow 0,
$$

соотношение (21) вытекает непосредственно из теоремы 3.

\section{3. Доказательства}

Доказательство теоремы 1. Введем обозначение $s_{i}$ для числа единиц в заполнении $i$-го регистра, $i=1, \ldots, r$ (в условиях теоремы это случайные величины). 
Лемма 1. Пусть $r \geq 2$. Тогда

$$
m_{1} \ldots m_{r}-2 \xi_{r}=\left(m_{1}-2 s_{1}\right) \ldots\left(m_{r}-2 s_{r}\right) .
$$

Доказательство леммы проводится индукцией по $r$. Сначала рассмотрим случай $r=2$. Из свойств сложения по модулю 2 следует, что

$$
\xi_{2}=\left(m_{1}-s_{1}\right) s_{2}+s_{1}\left(m_{2}-s_{2}\right)=\frac{m_{1} m_{2}}{2}-\frac{1}{2}\left(m_{1}-2 s_{1}\right)\left(m_{2}-2 s_{2}\right) .
$$

Значит,

$$
m_{1} m_{2}-2 \xi_{2}=\left(m_{1}-2 s_{1}\right)\left(m_{2}-2 s_{2}\right),
$$

и равенство (22) при $r=2$ доказано.

Выходную последовательность генератора с тремя регистрами можно рассматривать как выходную последовательность генератора с двумя регистрами, один из которых имеет длину $m_{1} m_{2}$ и заполнен выходной последовательностью генератора из первых двух регистров, а другим является третий регистр исходного генератора. Тогда согласно (23)

$$
\begin{gathered}
\left(m_{1} m_{2} m_{3}-2 \xi_{3}\right)=\left(m_{1} m_{2}-2 \xi_{2}\right)\left(m_{3}-2 s_{3}\right)= \\
=\left(m_{1}-2 s_{1}\right)\left(m_{2}-2 s_{2}\right)\left(m_{3}-2 s_{3}\right) .
\end{gathered}
$$

Аналогично проводится индуктивный переход при любом $r \geq 3$. Лемма доказана.

Из (22) следует, что

$$
\frac{2 \xi_{r}-m_{1} \ldots m_{r}}{\sqrt{m_{1} \ldots m_{r}}}=(-1)^{r-1} \frac{2 s_{1}-m_{1}}{\sqrt{m_{1}}} \ldots \frac{2 s_{r}-m_{r}}{\sqrt{m_{r}}} .
$$

Из (25) и симметричности относительно нуля распределений случайных величин, стоящих в скобках в правой части (25), вытекает (3). Теорема 1 доказана.

Доказательство следствия 1. Вычислим центральные моменты случайной величины $\xi_{r}$. Для этого преобразуем равенство (22) к виду

$$
\frac{m_{1} m_{2} \ldots m_{r}}{2}-\xi_{r}=2^{r-1}\left(\frac{m_{1}}{2}-s_{1}\right)\left(\frac{m_{2}}{2}-s_{2}\right) \ldots\left(\frac{m_{r}}{2}-s_{r}\right) .
$$

В условиях следствия величины $s_{1}, \ldots, s_{r}$ независимы между собой, а закон распределения случайной величины $s_{j}$ является биномиальным с параметрами $m_{j}$ и $1 / 2$. Поэтому применяя оператор математического ожидания к обеим частям последнего равенства, получаем первое из равенств (4). 
Так как распределение случайной величины $s_{j}$ симметрично относительно математического ожидания $m_{j} / 2$, то $\mathbf{E}\left(\frac{m_{j}}{2}-s_{j}\right)^{2 k-1}=0$. Отсюда следует (6). Производящая функция моментов для случайной величины $s_{j}-\frac{m_{j}}{2}$ равна

$$
\mathbf{E} e^{t\left(s_{j}-\frac{m_{j}}{2}\right)}=e^{-\frac{t m_{j}}{2}} \mathbf{E} e^{t s_{j}}=e^{-\frac{t m_{j}}{2}}\left(1+e^{t}\right)^{m_{j}} \frac{1}{2^{m_{j}}} .
$$

Поэтому

$$
\mathbf{E}\left(\frac{m_{j}}{2}-s_{j}\right)^{2 k}=\mu\left(m_{j}, 2 k\right) .
$$

Отсюда в силу независимости случайных величин $s_{1}, \ldots, s_{r}$ получаем (7) Формула (5) следует из (7), где надо положить $k=2$. Следствие 1 доказано.

Доказательство теоремы 2. Определим случайные величины

$$
\widetilde{s}_{i}=\frac{2 s_{i}-m_{i}}{\sqrt{m_{i}}}, \quad i=1, \ldots, r .
$$

Так как распределения дробей в правой части (25) симметричны относительно нуля, то из этого равенства следует, что

$$
\mathcal{L}\left(\widetilde{\xi}_{r}\right)=\mathcal{L}\left(\prod_{k=1}^{r} \widetilde{s}_{i}\right) .
$$

Перейдем к распределениям логарифмов абсолютных значений случайных величин, указанных в равенстве (26). Этот переход выражается в замене аргумента функций распределения $y$ на $e^{y}$. Кроме этого распределение произведения сменяется распределением суммы логарифмов.

Пусть

$$
\varsigma_{j}=\ln \left|\widetilde{s}_{j}\right|, \quad j=1, \ldots, r .
$$

Из (26) с учетом независимости сомножителей в правой части следует, что

$$
F_{\ln \left|\widetilde{\xi}_{r}\right|}(y)=F_{\varsigma_{1}} * \ldots * F_{\varsigma_{r}}(y),
$$

где знак * обозначает операцию свертки функций распределения.

Пусть случайные величины $\eta_{1}, \ldots, \eta_{r}$ независимы, имеют стандартное нормальное распределение и

$$
\delta_{i}=\ln \left|\eta_{i}\right|, \quad i=1, \ldots, r .
$$

Тогда

$$
F_{\sum_{i=1}^{r} \delta_{i}}(y)=F_{\delta_{1}} * \ldots * F_{\delta_{r}}(y)=F_{\delta_{1}}^{* r}(y) .
$$


В силу симметричности распределений имеем

$$
\begin{gathered}
F_{\left|\widetilde{\mid r}_{i}\right|}(y)=0, y<0, \quad F_{\left|\widetilde{\mid r}_{i}\right|}(y)=2 F_{\widetilde{s}_{i}}(y)-1, y \geq 0, \quad i=1, \ldots, r, \\
F_{\left|\widetilde{\xi}_{r}\right|}(y)=0, y<0, \quad F_{\left|\widetilde{\xi}_{r}\right|}(y)=2 F_{\widetilde{\xi}_{r}}(y)-1, y \geq 0, \\
F_{\left|\eta_{k}\right|}(y)=0, y<0, \quad F_{\left|\eta_{k}\right|}(y)=2 \Phi(y)-1, y \geq 0, \quad k=1, \ldots, r .
\end{gathered}
$$

Используя эти равенства, получаем

$$
\begin{gathered}
2 d\left(F_{\widetilde{s}_{i}}, \Phi\right)=2 \sup _{0 \leq y<\infty}\left|F_{\widetilde{s}_{i}}(y)-\Phi(y)\right|= \\
=\sup _{0 \leq y<\infty}\left|F_{\left|\widetilde{s}_{i}\right|}(y)-F_{\left|\eta_{i}\right|}(y)\right|=\sup _{-\infty \leq y<\infty}\left|F_{\widetilde{s}_{i} \mid}\left(e^{y}\right)-F_{\left|\eta_{i}\right|}\left(e^{y}\right)\right| .
\end{gathered}
$$

Кроме этого, $d(U * W, V * W) \leq d(U, V)$ для любых функций распределения $U, V$ и $W$. Поэтому из (27), (28) и (30) следует, что

$$
\begin{gathered}
d\left(F_{\ln \left|\widetilde{\xi}_{r}\right|}, F_{\delta_{1}}^{* r}\right)=d\left(F_{\varsigma_{1}} * \ldots * F_{\varsigma_{r}}, F_{\delta_{1}}^{* r}\right) \leq \\
\leq \sum_{k=0}^{r-1} d\left(F_{\varsigma_{1}} * \ldots * F_{\varsigma_{r-k}} * F_{\delta_{1}}^{* k}, F_{\varsigma_{1}} * \ldots * F_{\varsigma_{r-k-1}} * F_{\delta_{1}}^{* k+1}\right) \leq \\
\leq \sum_{k=1}^{r} d\left(F_{\varsigma_{k}}, F_{\delta_{k}}\right)=\sum_{k=1}^{r} d\left(F_{\left|\widetilde{s}_{k}\right|}, F_{\left|\eta_{k}\right|}\right)=2 \sum_{k=1}^{r} d\left(F_{\widetilde{S}_{k}}, \Phi\right) .
\end{gathered}
$$

Аналогично равенствам (30) выводится равенство

$$
2 d\left(F_{\tilde{\xi}_{r}}, \Phi_{r}\right)=\sup _{-\infty \leq y<\infty}\left|F_{\left|\widetilde{\xi}_{r}\right|}\left(e^{y}\right)-F_{\prod_{k=1}^{r}\left|\eta_{i}\right|}\left(e^{y}\right)\right|=d\left(F_{\ln \left|\widetilde{\xi}_{r}\right|}, F_{\delta_{1}}^{* r}\right) .
$$

Из этого равенства и (31) следует, что

$$
d\left(F_{\widetilde{\xi}_{r}}, \Phi_{r}\right)=\sum_{k=1}^{r} d\left(F_{\widetilde{s}_{k}}, \Phi\right) .
$$

Воспользуемся теперь тем, что расстояние в равномерной метрике между функциями распределения $F_{\widetilde{s}_{i}}(y)$ и $\Phi(y)$ допускает оценку (она следует из неравенства Берри - Эссеена)

$$
d\left(F_{\widetilde{s}_{i}}, \Phi\right) \leq \frac{C_{B E}}{\sqrt{m_{i}}}, \quad i=1,2, \ldots, r .
$$

Из (33) и (34) следует (9). Теорема 2 доказана. 
Доказательство следствия 2. В условиях следствия выражение в правой части неравенства (9) стремится к нулю. Отсюда вытекает (10). Следствие 2 доказано.

Доказательство теоремы 3. С учетом (34) можем записать

$$
\begin{gathered}
\left|F_{\varsigma_{j}}(x)-F_{\delta_{j}}(x)\right|=\left|F_{\widetilde{s}_{i}}\left(e^{x}\right)-F_{\widetilde{s}_{i}}\left(-e^{x}\right)-\Phi\left(e^{x}\right)+\Phi\left(-e^{x}\right)\right|= \\
=\left|F_{\widetilde{s}_{i}}\left(e^{x}\right)-\Phi\left(e^{x}\right)\right|+\left|F_{\widetilde{s}_{i}}\left(-e^{x}\right)-\Phi\left(-e^{x}\right)\right| \leq \frac{2 C_{B E}}{\sqrt{m_{j}}} .
\end{gathered}
$$

Пусть

$$
\widetilde{\delta}_{i}=\left(\delta_{i}-a\right) / \sigma \sqrt{r}, \quad \widetilde{\varsigma}_{i}=\left(\varsigma_{i}-a\right) / \sigma \sqrt{r} .
$$

Из (35) следует, что

$$
\left|F_{\widetilde{\varsigma}_{j}}(x)-F_{\widetilde{\delta}_{j}}(x)\right| \leq \frac{2 C_{B E}}{\sqrt{m_{j}}} .
$$

Используя (36), получим:

$$
\begin{gathered}
\left|F_{\sum_{j=1}^{r} \widetilde{\varsigma}_{j}}(x)-F_{\sum_{j=1}^{r} \widetilde{\delta}_{j}}(x)\right| \leq\left|F_{\widetilde{\varsigma}_{1}} * \ldots * F_{\varsigma_{r}}(x)-F_{\widetilde{\delta}_{1}} * \ldots * F_{\widetilde{\delta}_{r}}(x)\right| \leq \\
\leq \mid F_{\widetilde{\varsigma}_{1}} * \ldots * F_{\widetilde{\varsigma}_{r}}(x)-F_{\widetilde{\varsigma}_{1}} * \ldots * F_{\widetilde{\varsigma}_{r}-1} * F_{\widetilde{\delta}_{r}}(x)+ \\
+F_{\widetilde{\varsigma}_{1}} * \ldots * F_{\widetilde{\varsigma}_{r-1}} * F_{\widetilde{\delta}_{r}}(x)-F_{\widetilde{\delta}_{1}} * \ldots * F_{\widetilde{\delta}_{r}}(x) \mid \leq \\
\leq\left|F_{\widetilde{\varsigma}_{r}}(x)-F_{\tilde{\delta}_{r}}(x)\right|+\left|F_{\widetilde{\varsigma}_{1}} * \ldots * F_{\widetilde{\varsigma}_{r}-1}(x)-F_{\widetilde{\delta}_{1}} * \ldots * F_{\widetilde{\delta}_{r-1}}(x)\right| \leq \ldots \leq \\
\leq \sum_{i=1}^{r}\left|F_{\widetilde{\varsigma}_{i}}(x)-F_{\widetilde{\delta}_{i}}(x)\right| \leq 2 C_{B E} \sum_{i=1}^{r} \frac{1}{\sqrt{m_{i}}} .
\end{gathered}
$$

Согласно определениям $\mathbf{E} \delta_{i}=a, \mathbf{D} \delta_{i}=\sigma^{2}, \mathbf{E}\left|\delta_{i}-\mathbf{E} \delta_{i}\right|^{3}=c$, где величины $a, \sigma^{2}$ и $c$ определены равенствами (11), (12) и (13). Поэтому по неравенству Берри - Эссеена имеем

$$
\left|F_{\sum_{j=1}^{r} \tilde{\delta}_{j}}(x)-\Phi(x)\right| \leq C_{B E} \frac{\mathbf{E}\left|\delta_{j}-\mathbf{E} \delta_{j}\right|^{3}}{\left(\mathbf{D} \delta_{i}\right)^{3 / 2} \sqrt{r}}=C_{B E} \frac{c}{\sigma^{3} \sqrt{r}} .
$$

Таким образом, в силу (37) и (38)

$$
\begin{gathered}
\left|F_{\sum_{j=1}^{r} \widetilde{\varsigma}_{j}}(x)-\Phi(x)\right| \leq\left|F_{\sum_{j=1}^{r} \widetilde{\varsigma}_{j}}(x)-F_{\sum_{j=1}^{r} \widetilde{\delta}_{j}}(x)\right|+ \\
+\left|F_{\sum_{j=1}^{r} \widetilde{\delta}_{j}}(x)-\Phi(x)\right| \leq C_{B E}\left(2 \sum_{i=1}^{r} \frac{1}{\sqrt{m_{i}}}+\frac{c}{\sigma^{3} \sqrt{r}}\right) .
\end{gathered}
$$


Из формулы (25) следует, что

$$
\ln \left|m_{1} m_{2} \ldots m_{r}-2 \xi_{r}\right|=\sum_{j=1}^{r} \ln \left|m_{j}-2 s_{j}\right| .
$$

Преобразуем последнее равенство к виду

$$
\ln \left|m_{1} m_{2} \ldots m_{r}-2 \xi_{r}\right|-\frac{1}{2} \ln \left(m_{1} m_{2} \ldots m_{r}\right)=\sum_{j=1}^{r} \ln \frac{\left|m_{j}-2 s_{j}\right|}{\sqrt{m_{j}}} .
$$

Из (41) следует, что

$$
\ln \left|m_{1} m_{2} \ldots m_{r}-2 \xi_{r}\right|-\frac{1}{2} \ln \left(m_{1} m_{2} \ldots m_{r}\right)=\sum_{j=1}^{r} \varsigma_{j} .
$$

Значит,

$$
\ln \zeta_{r}=\frac{\ln \left|m_{1} m_{2} \ldots m_{r}-2 \xi_{r}\right|-\frac{1}{2} \ln \left(m_{1} m_{2} \ldots m_{r}\right)-r a}{\sigma \sqrt{r}}=\sum_{j=1}^{r} \widetilde{\varsigma}_{j} .
$$

Из (39) с учетом последнего равенства получаем (15):

$$
\left|F_{\zeta_{r}}(x)-\Upsilon(x)\right|=\left|F_{\ln \zeta_{r}}(\ln x)-\Phi(\ln x)\right| \leq C_{B E}\left(2 \sum_{i=1}^{r} \frac{1}{\sqrt{m_{i}}}+\frac{c}{\sigma^{3} \sqrt{r}}\right) .
$$

Теорема 3 доказана.

Доказательство следствия 3. Достаточно рассмотреть случай $x>0$. Из (29) следует, что в данном случае $F_{\widetilde{\xi}_{r}}(x)=\frac{1}{2}+\frac{1}{2} F_{\left|\widetilde{\xi}_{r}\right|}(x)$. С учетом этого равенства получаем

$$
\begin{gathered}
\left|F_{\widetilde{\xi}_{r}}(x)-\left(\frac{1}{2}+\frac{1}{2} \Phi\left(\frac{\ln x}{\sigma \sqrt{r}}-\frac{a}{\sigma} \sqrt{r}\right)\right)\right|= \\
=\left|\frac{1}{2} F_{\left|\widetilde{\xi}_{r}\right|}(x)-\frac{1}{2} \Phi\left(\frac{\ln x}{\sigma \sqrt{r}}-\frac{a}{\sigma} \sqrt{r}\right)\right|=\frac{1}{2}\left|\mathbf{P}\left\{\widetilde{\xi}_{r} \mid<x\right\}-\Upsilon\left(e^{-\frac{a}{\sigma} \sqrt{r}} x^{\frac{1}{\sigma \sqrt{r}}}\right)\right|= \\
=\frac{1}{2}\left|\mathbf{P}\left\{\zeta_{r}<e^{-\frac{a}{\sigma} \sqrt{r}} x^{\frac{1}{\sigma \sqrt{r}}}\right\}-\Upsilon\left(e^{-\frac{a}{\sigma} \sqrt{r}} x^{\frac{1}{\sigma \sqrt{r}}}\right)\right| .
\end{gathered}
$$

Из последнего равенства и (15) имеем (17) для $x>0$. В силу симметричности распределения случайной величины $\widetilde{\xi}_{r}$ оценка (18) выполнена и при $x<0$. Следствие 3 доказано. 
Доказательство следствия 4. В условиях следствия правая часть (17) стремится к нулю. Найдем такое число $x_{\alpha}$, для которого $F_{\widetilde{\xi}_{r}}\left(x_{\alpha}\right) \rightarrow \alpha$.

Обозначим $\frac{\ln x}{\sigma \sqrt{r}}-\frac{a}{\sigma} \sqrt{r}=u$. Тогда $x=x_{u}=e^{u \sigma \sqrt{r}+a r}$. Выберем величину $u$ так, чтобы $\frac{1}{2}+\frac{1}{2} \Phi(u)=\alpha$, т. е. $\Phi(u)=2 \alpha-1$ и $u=u_{2 \alpha-1}$. При таком выборе параметров из (17) имеем $F_{\widetilde{\xi}_{r}}\left(x_{u_{2 \alpha-1}}\right) \rightarrow \alpha$. Последнее соотношение эквивалентно тому, что

$$
\mathbf{P}\left\{\widetilde{\xi}_{r}<e^{u_{2 \alpha-1} \sigma \sqrt{r}+a r}\right\} \rightarrow \alpha .
$$

Тем самым, соотношение (18) доказано. Из (18) и симметричности распределения случайной величины $\widetilde{\xi}_{r}$ вытекает соотношение (19). Следствие 4 доказано.

Доказательство теоремы 4. Используя наши определения, независимость заполнений регистров и равенство (22), получаем

$$
\frac{\ln \left|\widetilde{\xi}_{r}\right|-\mathbf{E} \ln \left|\widetilde{\xi}_{r}\right|}{\sqrt{\mathbf{D} \ln \left|\widetilde{\xi}_{r}\right|}}=\sum_{k=1}^{r} \frac{\varsigma_{k}-\mathbf{E} \varsigma_{k}}{\sqrt{\sum_{j=1}^{r} \mathbf{D} \varsigma_{j}}},
$$

где, напомним,

$$
\varsigma_{j}=\ln \left|\widetilde{s}_{j}\right|=\ln \left|\frac{2 s_{j}-m_{j}}{\sqrt{m_{j}}}\right|, \quad j=1, \ldots, r .
$$

Так как все числа $m_{1}, \ldots, m_{r}$ четны и взаимно просты, то моменты

$$
\mathbf{E} \varsigma_{j}^{k}=\frac{1}{2^{m_{j}}} \sum_{i=0}^{m_{j}} C_{m_{j}}^{i} \ln ^{k}\left(\frac{\left|2 i-m_{j}\right|}{\sqrt{m_{j}}}\right)
$$

конечны при всех $k=1,2, \ldots$ Более того, из свойств биномиального распределения, которому подчинены случайные величины $s_{j}$, и неравенств $3 \leq$ $\leq m_{1}<m_{2}<\ldots$ следует, что при $j \rightarrow \infty$ моменты $\mathbf{E} \varsigma_{j}^{k}$ сходятся к соответствующим моментам логарифма модуля случайной величины со стандартным нормальным распределением (которые тоже конечны). Поэтому все эти моменты равномерно (по $j$ ) ограничены. То же самое, разумеется, относится и к центральным моментам.

Из этого, в частности, следует, что при $r \rightarrow \infty$

$$
\mathbf{D} \ln \left|\widetilde{\xi}_{r}\right|=\sum_{j=1}^{r} \mathbf{D} \varsigma_{j}=r \sigma^{2}(1+o(1))
$$

(величина $\sigma^{2}$ определена в (12)). 
К распределению суммы (42) независимых случайных величин с равномерно ограниченными моментами применим неравенство Берри - Эссеена, но для неодинаково распределенных случайных величин:

$$
\left|\mathbf{P}\left\{\frac{\ln \left|\widetilde{\xi}_{r}\right|-\mathbf{E} \ln \left|\widetilde{\xi}_{r}\right|}{\sqrt{\mathbf{D} \ln \left|\widetilde{\xi}_{r}\right|}}<x\right\}-\Phi(x)\right|<\frac{C}{\sqrt{\mathbf{D} \ln \left|\widetilde{\xi}_{r}\right|}},
$$

где $C<\infty$.

Из (44) и (43) вытекает соотношение

$$
\mathbf{P}\left\{\frac{\ln \left|\widetilde{\xi}_{r}\right|-\mathbf{E} \ln \left|\widetilde{\xi}_{r}\right|}{\sigma \sqrt{r}}<x\right\} \rightarrow \Phi(x), \quad r \rightarrow \infty .
$$

В свою очередь, из (45) следует (20). Теорема 4 доказана.

Авторы благодарны А. М.Зубкову и А.М.Шойтову за полезные замечания.

\section{Список литературы}

1. Pohl P. Description of MCV, a pseudo-random number generator // Scand. Actuarial J. - 1976. - № 1. - P. 1-14.

2. Петров В. В. Суммы независимых случайных величин. - М.: Наука, 1972.

3. Тюрин И. С. Уточнения остаточного члена в теореме Ляпунова // Теория вероятн. и применен. - 2011. - Т. 56. Вып. 3. - С. 808-811. 